University of Wollongong

Research Online

Australian Institute for Innovative Materials -

Papers

Australian Institute for Innovative Materials

$1-1-2018$

s-p orbital hybridization: a strategy for developing efficient photocatalysts with high carrier mobility

Zhongfei Xu

Beihang University, Ministry of Education, xuzfei@buaa.edu.cn

Kang Xu

Beihang University, Ministry of Education

Haifeng Feng

University of Wollongong, Beihang University, hf533@uowmail.edu.au

Yi Du

University of Wollongong, Beihang University, ydu@uow.edu.au

Weichang Hao

Beihang University, Ministry of Education, whao@buaa.edu.cn

Follow this and additional works at: https://ro.uow.edu.au/aiimpapers

Part of the Engineering Commons, and the Physical Sciences and Mathematics Commons

Research Online is the open access institutional repository for the University of Wollongong. For further information contact the UOW Library: research-pubs@uow.edu.au 


\title{
s-p orbital hybridization: a strategy for developing efficient photocatalysts with high carrier mobility
}

\author{
Abstract \\ Photocatalysis has not only invigorated the field of energy conversion materials, but also is leading to \\ bright prospects for application in the environmental purification field [1]. Akira Fujishima and Kenichi \\ Honda [2] first reported photocatalytic water splitting on a TiO2 semiconductor electrode under ultraviolet \\ (UV) light in 1972. In semiconductor photocatalysts, electrons are excited from valence band maximum \\ (VBM) to conduction band minimum (CBM) under light irradiation, and then trigger the photocatalytic \\ process [3]. Considering solar-light-driven photocatalysis, semiconductor photocatalysts should possess \\ a narrow band gap and appropriate band positions [4]. It was also found that photoinduced charge \\ generation, separation, and transportation determine activities of semiconductor photocatalysts. High \\ mobility of charge carriers facilitates these processes, which can be achieved in the photocatalysts with \\ highly dispersive bands, because their effective masses of charge carriers are small. Usually, the \\ antibonding hybridization/coupling is predominantly responsible for the band dispersion, especially for \\ oxides. For example, Sn-5s/O- 2p anti-bonding coupling in VBM of Sn2+ oxides, Cu-3d/O-2p anti-bonding \\ coupling in VBM of $\mathrm{Cu}+$ oxides and anti-bonding coupling in CBM of most of semiconductors [5-9]. \\ Especially, s-p orbital hybridization is found to improve the performance of photocatalysts by affecting \\ their band structures [10,11].

\section{Disciplines \\ Engineering | Physical Sciences and Mathematics}

\section{Publication Details} \\ Xu, Z., Xu, K., Feng, H., Du, Y. \& Hao, W. (2018). s-p orbital hybridization: a strategy for developing efficient \\ photocatalysts with high carrier mobility. Science Bulletin, 63 (8), 465-468.
}




\section{Science Bulletin}

s-p orbital hybridization: a strategy for developing efficient photocatalysts with high carrier mobility

Xu Zhong-Fei, Xu Kang, Feng Hai-Feng, Du Yi and Hao Wei-Chang

Citation: Science Bulletin 63, 465 (2018); doi: 10.1016/j.scib.2018.02.020

View online: http://engine.scichina.com/doi/10.1016/j.scib.2018.02.020

View Table of Contents:http://engine.scichina.com/publisher/scp/journal/SB/63/8

Published by the Science China Press

Articles you may be interested in 


\title{
s-p orbital hybridization: a strategy for developing efficient photocatalysts with high carrier mobility
}

\author{
Zhong-Fei $\mathrm{Xu}^{\mathrm{a}, \mathrm{b}}$, Kang $\mathrm{Xu}^{\mathrm{a}, \mathrm{b}}$, Hai-Feng Feng ${ }^{\mathrm{b}, \mathrm{c}}$, Yi Du ${ }^{\mathrm{b}, \mathrm{c}}$, Wei-Chang Hao ${ }^{\mathrm{a}, \mathrm{b}, *}$ \\ a Department of Physics and Key Laboratory of Micro-nano Measurement, Manipulation and Physics, Ministry of Education (MOE), Beihang University, Beijing 100191, China \\ ${ }^{\mathrm{b}}$ BUAA-UOW Joint Research Centre, Beihang University, Beijing 100191, China \\ ${ }^{\mathrm{c}}$ Institute for Superconducting and Electronic Materials, University of Wollongong, Wollongong, NSW 2522, Australia
}

A R T I C L E I N F O

Article history:

Received 22 December 2017

Received in revised form 11 February 2018

Accepted 24 February 2018

Available online 2 March 2018 (c) 2018 Science China Press. Published by Elsevier B.V. and Science China Press. All rights reserved.
Photocatalysis has not only invigorated the field of energy conversion materials, but also is leading to bright prospects for application in the environmental purification field [1]. Akira Fujishima and Kenichi Honda [2] first reported photocatalytic water splitting on a $\mathrm{TiO}_{2}$ semiconductor electrode under ultraviolet (UV) light in 1972. In semiconductor photocatalysts, electrons are excited from valence band maximum (VBM) to conduction band minimum (CBM) under light irradiation, and then trigger the photocatalytic process [3]. Considering solar-light-driven photocatalysis, semiconductor photocatalysts should possess a narrow band gap and appropriate band positions [4]. It was also found that photoinduced charge generation, separation, and transportation determine activities of semiconductor photocatalysts. High mobility of charge carriers facilitates these processes, which can be achieved in the photocatalysts with highly dispersive bands, because their effective masses of charge carriers are small. Usually, the antibonding hybridization/coupling is predominantly responsible for the band dispersion, especially for oxides. For example, Sn-5s/O$2 \mathrm{p}$ anti-bonding coupling in VBM of $\mathrm{Sn}^{2+}$ oxides, $\mathrm{Cu}-3 \mathrm{~d} / \mathrm{O}-2 \mathrm{p}$ anti-bonding coupling in $\mathrm{VBM}$ of $\mathrm{Cu}^{+}$oxides and anti-bonding coupling in CBM of most of semiconductors [5-9]. Especially, s-p orbital hybridization is found to improve the performance of photocatalysts by affecting their band structures [10,11]. It has been widely recognized that band gaps and band positions can be optimized by adjusting the hybridization of electrons in the band structure by introducing $\mathrm{s}$ or $\mathrm{p}$ orbitals from the doping elements [12]. Inspired by this, s-p orbital hybridization has attracted great attention recently. It has been reported that the dispersive and broad hybridized In 5s5p orbitals in $\mathrm{MIn}_{2} \mathrm{O}_{4}(\mathrm{M}=\mathrm{Ca}$, $\mathrm{Sr}), \mathrm{Sr}_{0.93} \mathrm{Ba}_{0.07} \mathrm{In}_{2} \mathrm{O}_{4}$, and $\mathrm{AInO}_{2}(\mathrm{~A}=\mathrm{Li}, \mathrm{Na})$ are neccessary to induce its high dispersive band structure for photo-excited elec-

\footnotetext{
* Corresponding author.

E-mail address: whao@buaa.edu.cn (W.-C. Hao).
}

tron transfers with small recombination rate [13]. It is also found that $\mathrm{O} 2 \mathrm{p}$ and $\mathrm{Ag}^{+} \mathrm{s}$ or $\mathrm{Zn}^{+} \mathrm{s}$ orbitals in photocatalysts can improve the mobility of photo-excited charge carriers because of the greatly dispersed s orbitals [14]. Ag 5s and $\mathrm{Ag} 5 \mathrm{p}$ states of $\mathrm{Ag}_{3} \mathrm{PO}_{4}$ in the CBM lead to a more dispersive energy band and increase the light absorption ability by narrowing the band gap [15]. It is indeed adopted that the s-p orbital hybridization is an efficient strategy for endowing active photocatalysts with high carrier mobility, yet no investigations on the mechanism behind this phenomenon have been addressed to date. Understanding the roles of s-p orbital hybridization is, therefore, essential for the rational optimization of highly efficient photocatalysts. In this paper, we have studied the origins of dispersive energy bands based on basic quantum mechanics and valence bond theory. Additional evidence for $s-p$ orbital hybridization is explored, with the aim of obtaining smaller effective mass. Finally, three strategies are proposed for designing active photocatalysts with high carrier mobility.

The success of describing high carrier mobility using small effective mass with the dispersive energy band is well known for semiconductors. Effective mass is calculated by the following equation,

$m^{*}=\frac{\hbar^{2}}{\frac{\partial^{2} E(k)}{\partial k^{2}}}$,

where $m^{*}$ is the effective mass, $\hbar$ is the reduced Planck constant, and $E(k)$ is the energy of an electron at wave vector $k$ in that particular band. Eq. (1) demonstrates that the effective mass of a semiconductor is obtained by fitting its actual $E(k)-k$ diagram around the CBM or VBM by a parabola [16]. Consequently, a dispersive energy band is desired to provide smaller effective mass, which relates to higher charge carrier mobility in semiconductors [17]. The s-p orbital hybridization, which can produce more dispersive energy band because of their large overlap between orbitals, is investigated here 
in more detail. To investigate whether s-p hybridization can render an explanation for this behavior, the origins of dispersive energy bands are first considered. Then, the reasons for choosing s-p hybridization are addressed. Finally, the strategies for using s-p hybridization in searching for materials with high carrier mobility are proposed.

According to the energy band theory, in the single isolated atom, a single particle orbital has a definite energy which refers to the energy level, as shown at the top of Fig. S1a (online). When two or more isolated atoms are close enough, electrons in the outermost orbital will experience an attractive force causing them to interact with their nearest neighbors, so that these electrons are shared. The energy of shared electrons will change according to Pauli exclusion principle. Ultimately, a continuous energy band is formed as the number of atoms increases, as is exhibited at the bottom of Fig. S1a (online). Whereas, the interaction between inner-shell electrons of nearest-neighbored atoms is much weaker than outermost electrons. Thus, the energy band formed by the outermost electron is broader than those of the inner-shell electrons, as reflected in Fig. S1b (online). In the energy band theory, the band structure of a periodic system is determined by specifying the Bloch functions $\psi(r)$, which are constructed from the electron orbitals based on the reciprocal vector $k$ inside the Brillouin zone (BZ) and the energy eigenvalue $E_{n}(k)$. The energy eigenvalues of electrons are obtained by solving the Schrödinger equation

$H \cdot \psi(r)=E_{n} \cdot \psi(r)$

$E_{n}(k)=E_{n}{ }^{m}-A_{n}-J_{n} \sum_{r} \mathrm{e}^{\mathrm{i} k \cdot r}$,

where $E_{n}{ }^{m}$ is the atomic energy level of a single particle orbital $m$ at ground state, $A_{n}$ is the change in $E_{n}{ }^{m}$ when influenced by the periodic potential field of the crystal structure, which is a minimal value based on the perturbation theory, $J_{n}$ is the overlap integral of the electron orbital $m$ between atoms, $r$ is the lattice vector, and $k$ is the wave vector of wave function. Differences between maximum and minimum eigenvalue would be confined to the region dominated by $J_{n}$, possibly evident as the width of the energy band for the single particle orbitals $m$. Therefore, the overlap of these orbitals intuitively accommodates widths of energy bands, which is a manifestation of the band dispersion. Consequently, a large overlap between single particle orbitals with same or similar energy of neighboring atoms can create a broad energy band and result in a dispersive band structure.

In general, carrier mobility is degenerate with respect to the value of effective mass in the VBM or CBM. Therefore, valence electrons in the outermost orbitals, whose energy band is located in the vicinity of the Fermi level, are concerned. Here we should mention the origin of band gap, which is analogous to the formation of band broadening. As pictured in Fig. S1c (online), the overlapping of different energy bands introduces a repulsive interaction between them, which produces a band gap in materials by lowering the lower band and raising the higher band. It is worth emphasizing that the band gap reflects the overlapping of different energy bands while band broadening is contributed by the overlapping of particle orbital with same or similar energy. These behaviors are summarized in Fig. S1d (online) [18], taking carbon atoms as the example. Two isolated $C$ atoms display the same two energy levels designated as $2 \mathrm{~s}$ and $2 \mathrm{p}$ (at point I), which broaden into energy bands as the distance between atoms is reduced. The widths of both energy bands originating from $2 \mathrm{~s}$ and $2 \mathrm{p}$ are increased, derived from the larger overlap of the relevant electrons (I-O). In the presence of overlap between the thus-formed energy bands for $2 \mathrm{~s}$ and $2 \mathrm{p}$, a band gap appears (where point $\mathrm{C}$ represents the actual situation in diamond). In order to give an accurate description for the complex real crystal structure, point $\mathrm{C}$ is discussed
(Fig. S2 online). Valence electrons of C $2 \mathrm{~s}$ and $\mathrm{C} 2 \mathrm{p}$ in the unit cell have already overlapped in the diamond lattice. Otherwise, the hybridized s-p orbital will contribute to both the VB and the CB. According to the analysis above, the band width preserved by the valence electrons is determined by $s, p$, and $s-p$ orbitals, while the band gap is ascribed to the interaction between them.

Since carrier mobility is proportional to dispersion of energy bands, constructing a large overlap of orbitals through rational alignment of valence electrons is then expected to result in high carrier mobility. s-p hybridization, a possible electron configuration for a dispersive energy band, is described here. To understand this, it is instructive to analyze the orbital wave function which gives the distribution of the kinematic states of electrons in real space. The square root of the orbital wave function is the distribution function of electron probability, which is given in Fig. S3a for s, $\mathrm{p}$, and d orbitals [19]. Their relative sizes correspond to the binding ability of orbitals. By setting that value of the s orbital as 1 , it is $\sqrt{3}$ and $\sqrt{5}$ for the p orbital and d orbital, respectively [20]. This leads us to conclude that the relative binding ability decreases in the order of s, p, and d orbitals. In addition, s-s and s-p hybridization preferentially yield the strongest type of interaction, $\sigma$ bonding, which arises from considerable overlap between orbitals (Fig. S3b online). Hence, the strong s-p hybridization is implicitly assumed to be the most favorable configuration for broadening and dispersing the energy band in $k$ space, which is expected to harvest smaller effective mass and higher carrier mobility in semiconductors. Otherwise, the anti-bonding s-p orbital hybridization in CBM (or VBM) is also a good strategy to develop efficient photocatalysts with a high electron (or hole) mobility. Promising candidates for elements of these materials in the periodic table are highlighted with the black frame in Fig. 1a. Cation elements Tl, $\mathrm{Pb}, \mathrm{Po}$, and At are not included because of their instability and toxicity. With 9 cation and 16 anion elements selected, there are 144 combinations of elements possible for binary materials.

It has been verified that $s-p$ orbital hybridization is beneficial to energy-band dispersion, which will lower the effective mass and increase the carrier mobility. Motivated by these considerations, we have developed three strategies based on s-p orbital hybridization according to Eq. (3), as shown in the schematic diagram in Fig. 1b. Firstly, $E_{n}{ }^{m}$ reflects the intrisic property of atoms. Elements with rich $s$ and $p$ valence electrons should be selected, such as Pblock elements $\left(\mathrm{s}^{2} \mathrm{p}^{x}\right)$ or $\mathrm{d}^{10}$ metal cations (for example, Ag $\left(4 d^{10} 5 s^{1}\right)$ and $\left.\mathrm{Zn}\left(3 \mathrm{~d}^{10} 4 \mathrm{~s}^{2}\right)\right)$. Secondly, $A_{n}$ reflects the influence of crystal potential field. The close packed crystal structure, such as the diamond structure, is also helpful to achieve larger overlap between valence electrons. Finally, $J_{n}$ reflects the interaction between valence electrons of atoms. Larger sizes of atoms are better than smaller one due to their greater opportunity for overlapping with each other. However, the last one should not be overstated because compounds with too large-size atoms may lead to very small band gap which will degrade the performance of photocatalysts.

Some typical s-p hybridization materials are selected to analyze the relationship between band decomposition and effective mass. More insight can be gained by calculating the electron structures of typical photocatalysts that are composed of the candidate elements. Calculation methods are given in the Supplementary data (online). We group them into three categories according to their elements and band composition, as indicated by s-p, s-p-d, and Bi-based species. Summarized orbital compositions of the CBM and VBM (which are depicted in terms of their partial density of states (PDOS) in Fig. S5 online) for these photocatalysts are displayed in Table S1 and Fig. S4 (online). The calculation results imply that the CBM and VBM of these materials are dominated by $s-p$ or $p$ orbitals, except for the s-p-d species. The calculated 
(a)

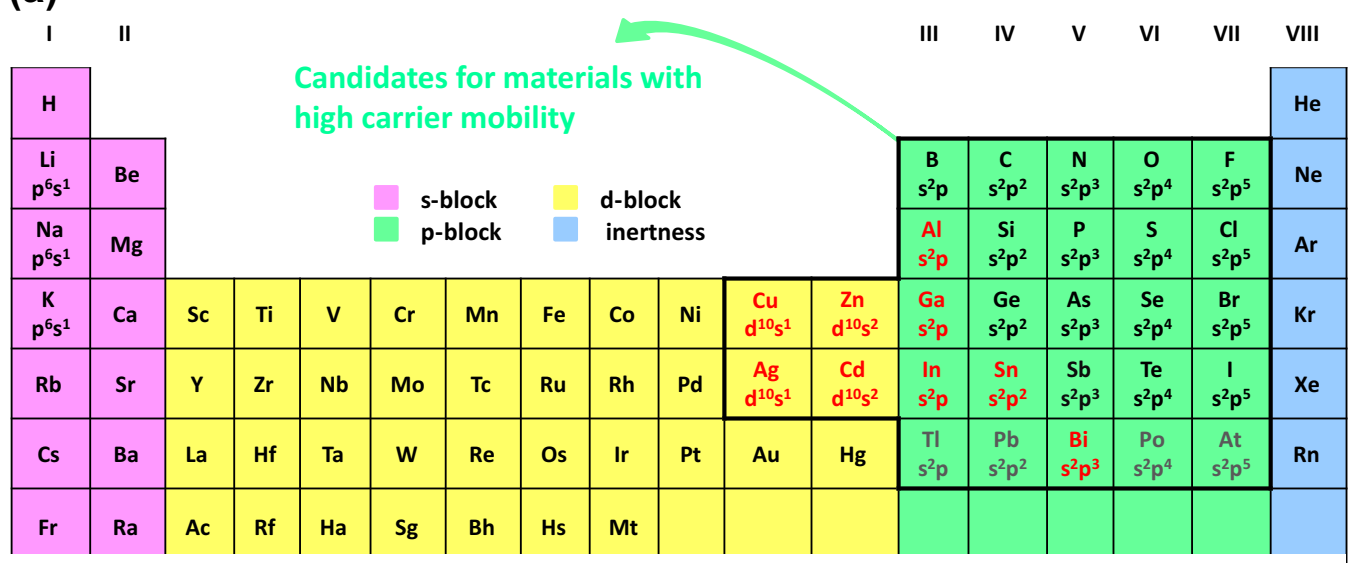

(b)

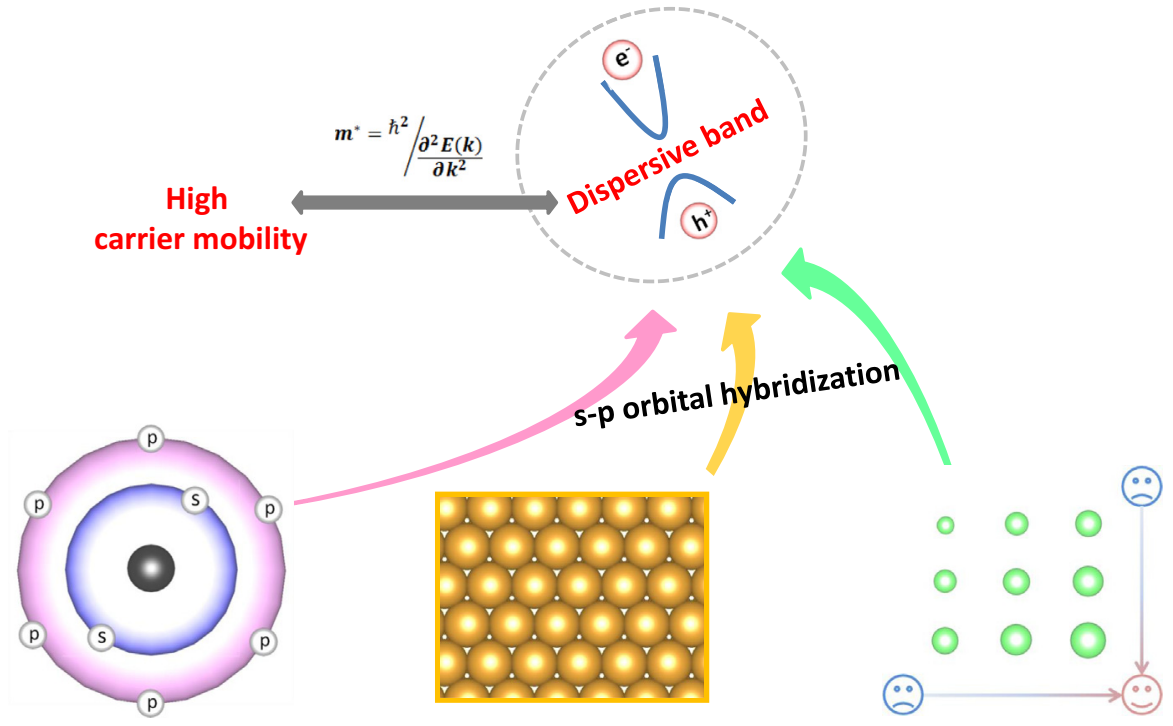

s and $p$ orbitals

Close packing crystal

Large size of atoms

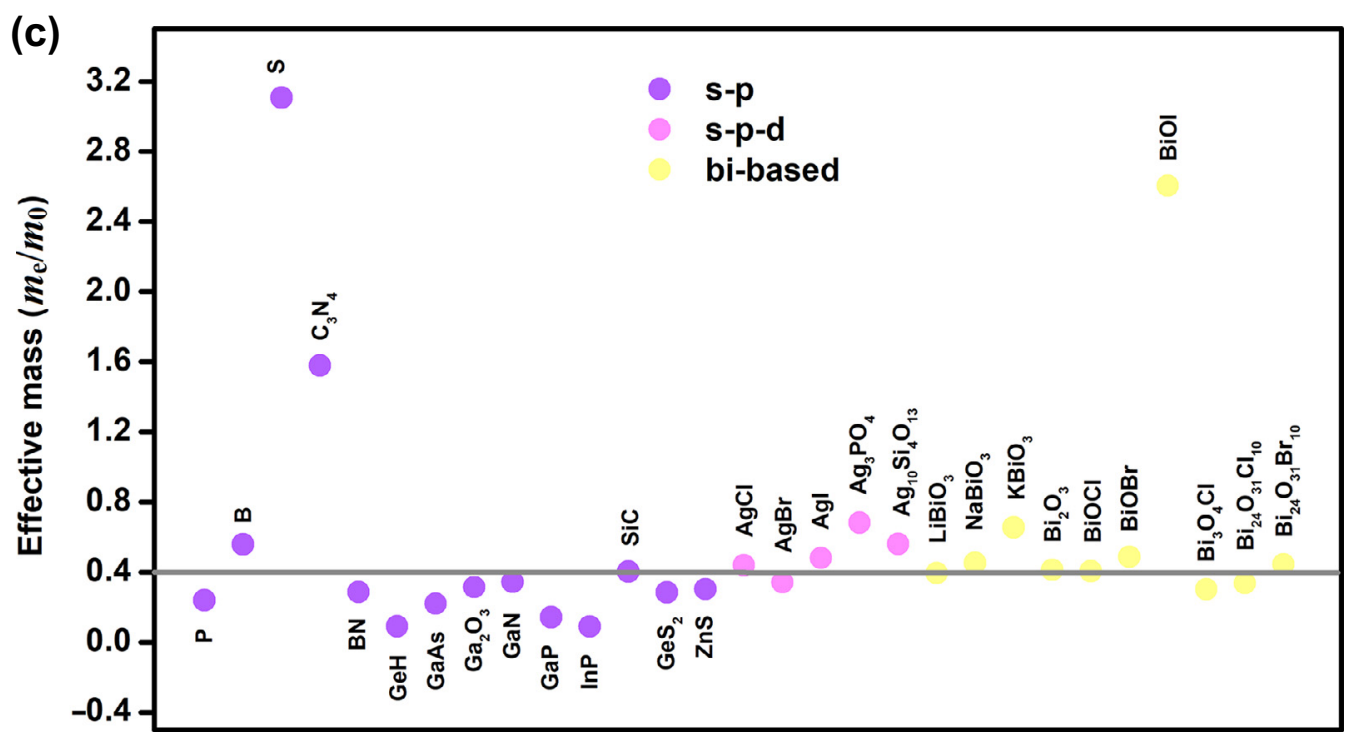

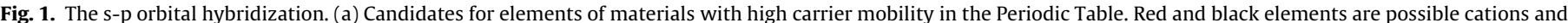

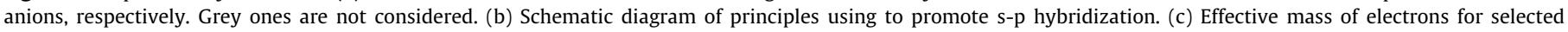
photocatalysts. Purple, pink, and yellow balls are s-p, s-p-d, and Bi-based species.

band structures are given in Fig. S6 (online) and effective masses of selected photocatalysts are listed in $\mathrm{T}_{2} \mathrm{Tble}_{2} \mathrm{~S}_{2}$ (online), elucidating the principles for s-p orbital hybridization by having the smallest effective mass at the $\mathrm{CBM}\left(\mathrm{Fig}_{5} / \mathrm{A}\right)$ ). The value of effective mass 
$0.4 m_{0}$ ( $m_{0}$ is the electron mass) is set as a criterion to identify the electron mobility. The results clearly suggest that effective mass of most of the s-p materials is mostly smaller than those of s-p-d and Bi-based materials. This phenomenon is in good agreement with s-p orbital hybridization strategy that a smaller effective mass results from stronger hybridization between $\mathrm{s}$ and $\mathrm{p}$ orbitals. As shown in Fig. S4 (online), both the VBM and the CBM of the elements $\mathrm{P}, \mathrm{B}$, and $\mathrm{S}$ are comprised of $\mathrm{s}$ and $\mathrm{p}$ orbitals. The VBM and CBM of BN consists of B-p and N-p orbitals, while the CBM of $\mathrm{C}_{3} \mathrm{~N}_{4}$ only originates from C-p and N-p orbitals and the VBM from $\mathrm{N}-\mathrm{p}$ and $\mathrm{N}-\mathrm{s}$ orbitals. Other materials in s-p species have similar components, with their CBM coming from s-p orbitals, while hybridized p orbitals are observed in the VBM. Efficient cooperation and hybridization of $\mathrm{s}$ and $\mathrm{p}$ orbitals may give a good explanation for their higher carrier mobility. In the case of s-p-d species, although $\mathrm{d}$ orbitals are observed in the VBM, the participation of $\mathrm{p}$ orbitals in the VBM and the existence of s-p hybridization in the CBM support their good photocatalytic activity. In Bi-based materials, the CBM of $\mathrm{MBiO}_{3}(\mathrm{M}=\mathrm{Li}, \mathrm{Na}, \mathrm{K})$ compounds consists of s-p orbitals, while p orbitals mainly contribute to the $\mathrm{VBM}$. $\mathrm{BiOX}(\mathrm{X}=\mathrm{Cl}, \mathrm{Br}, \mathrm{I})$ only has $p$ orbitals in both the CBM and the VBM. Other Bi-based materials all have similar constituents in the CBM (p orbitals) and VBM (s-p orbitals). The lower carrier mobility of Bi-based species may be related to their multiple-element nature and non-compact lattice, which lead to a large effective mass. In all of these selected materials, GeH, GaAs, GaP and InP have the smallest effective masses which are located in the area of s-p species. The small effective mass also has a close relationship to their close packed structures and bigger sizes of atoms, which work together with s-p orbital hybridization for creating a larger overlap between atoms and obtaining smaller effective mass. According to our results, $\mathrm{GeH}, \mathrm{GaAs}, \mathrm{GaP}, \mathrm{InP}, \mathrm{P}, \mathrm{BN}, \mathrm{GeS}_{2}$, and $\mathrm{ZnS}$ are indicated as the promising candidates for photocatalysts with high carrier mobility.

In conclusion, we have described the origins of dispersive energy bands to determine the decisive factor for high charge carrier mobility. A dispersive energy band indicates a small effective mass in VBM or CBM with expected high carrier mobility. It is estimated that large overlap between valence electrons plays the primary role in large energy band dispersion. s-p orbital hybridization is observed to possibly create larger overlap because of its stronger binding ability. Three strategies, which are helpful for designing active photocatalysts with high carrier mobility, are proposed based on s-p orbital hybridization. Firstly, elements with rich s and $\mathrm{p}$ electrons are required to obtain well cooperating s-p orbital hybridization. Secondly, close packed structures are expected to gain stronger overlap between valence electrons. Thirdly, larger sizes of atoms are benefit to achieve smaller effective mass. Based on the results of DOS and effective mass, GeH, GaAs, GaP, InP, P, BN, $\mathrm{GeS}_{2}$, and $\mathrm{ZnS}$ are indicated as the promising candidates for photocatalysts with high carrier mobility.

\section{Conflict of interest}

The authors declare that they have no conflict of interest.

\section{Acknowledgments}

This work was financially supported by the National Natural Science Foundation of China (51672018, 51472016 and 51272015), and Fundamental Research Fund for Centre University. Yi Du thank the Australian Research Council Discovery Project (DP170101467) and University of Wollongong AIIM for Gold Grant

\section{Appendix A. Supplementary data}

Supplementary data associated with this article can be found, in the online version, at https://doi.org/10.1016/j.scib.2018.02.020.

\section{References}

[1] Kudo A. Development of photocatalyst materials for water splitting. Int J Hydro Energy 2006;31:197-202.

[2] Fujishima A, Honda K. Electrochemical photolysis of water at a semiconductor electrode. Nature 1972;238:37-8.

[3] Hoffmann MR, Martin ST, Choi W, et al. Environmental applications of semiconductor photocatalysis. Chem Rev 1995;95:69-96.

[4] Takashi H, Kubota J, Domen K. Recent advances in semiconductors for photocatalytic and photoelectrochemical water splitting. Chem Soc Rev 2014;43:7520-35.

[5] Hautier G, Miglio A, Ceder G, et al. Identification and design principles of low hole effective mass p-type transparent conducting oxides. Nat Commun 2013;4:2292.

[6] Walsh A, Watson GW. Electronic structures of rocksalt, litharge, and herzenbergite SnO by density functional theory. Phys Rev B 2004;70:235114.

[7] Li YW, Singh DJ, Du MH, et al. Design of ternary alkaline-earth metal Sn(II) oxides with potential good p-type conductivity. J Mater Chem C 2016;4:4592-9.

[8] Xu QL, Li YW, Zhang LJ, et al. (2017) Sn(II)-containing phosphates as optoelectronic materials. Chem Mater 2017;29:2459-65.

[9] Gillen R, Robertson J. Band structure calculations of $\mathrm{CuAlO}_{2}, \mathrm{CuGaO}_{2}$, $\mathrm{CuInO}_{2}$, and $\mathrm{CuCrO}_{2}$ by screened exchange. Phys Rev B 2011;84:035125.

[10] Wu DH, Zhang X, Jing $Y$, et al. Towards visible-light water splitting photocatalysts: band engineering of two-dimensional $\mathrm{A}_{5} \mathrm{~B}_{4} \mathrm{O}_{15}$ perovskites. Nano Energy 2016;28:390-6.

[11] Zhang X, Zhao XD, Wu DH, et al. MnPSe 3 Monolayer: a promising 2D visible-light photohydrolytic catalyst with high carrier mobility. Adv Sci 2016;3:1600062.

[12] Asahi RY, Morikawa TA, Ohwaki T, et al. Visible-light photocatalysis in nitrogen-doped titanium oxides. Science 2001;293:269-71.

[13] Sato J, Nishiyama H, Inoue Y. Photocatalytic activity for water decomposition of indates with octahedrally coordinated $\mathrm{d}^{10}$ configuration. II. Influences of preparation conditions on activity. J Phys Chem B 2003;107:7965-9.

[14] Tang JW, Ye JH. Photocatalytic and photophysical properties of visible-lightdriven photocatalyst $\mathrm{ZnBi}_{12} \mathrm{O}_{20}$. Chem Phys Lett 2005;410:104-7.

[15] Liu JJ, Fu XL, Chen SF, et al. Electronic structure and optical properties of $\mathrm{Ag}_{3} \mathrm{PO}_{4}$ photocatalyst calculated by hybrid density functional method. Appl Phys Lett 2011;99:191903.

[16] Umezawa N, Ouyang SX, Ye JH. Theoretical study of high photocatalytic performance of $\mathrm{Ag}_{3} \mathrm{PO}_{4}$. Phys Rev B 2011;83:035202.

[17] Maruyama Y, Irie H, Hashimoto K. Visible light sensitive photocatalyst, delafossite structured $\alpha-\mathrm{AgGaO}_{2}$. J Phys Chem B 2006;110:23274-8.

[18] Yan SS. Fundamentals of solid state physics. 1st ed. Beijing: Peking University; 2000.

[19] Pauling L. The nature of the chemical bond. 3rd ed. Ithaca: Cornell University; 1960.

[20] Hultgren R. Equivalent chemical bonds formed by s, p, and d eigenfunctions. Phys Rev 1932;40:891.

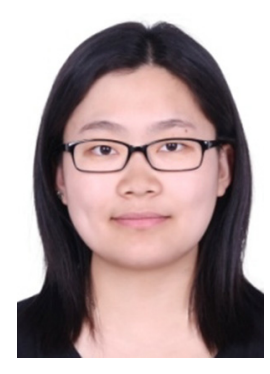

Zhongfei $\mathrm{Xu}$ is studying at Beihang University. Her research interests include first-principle calculations of electrochemical activity and electronic/phononic properties of oxides under pressure.

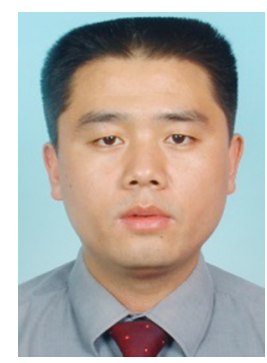

Weichang Hao received his Ph.D. degree from Lanzhou University in 2003. Then he joined in Beihang University. He was appointed as a full Professor in 2013. His research interests include the electronic structures of oxides, applications of oxides in environment and energy, and 2D nanomaterial devices. 